\title{
Eventos de massa, desastres e Saúde Pública
}

\author{
Mass gatherings, disasters and public health
}

Camilla Figueiredo de Castro ${ }^{1}$

Dayane Carla Menezes Simões ${ }^{1}$

Elizabete Vianna Delamarque ${ }^{1}$

Vera Lucia Edais Pepe ${ }^{1}$

${ }^{1}$ Departamento de Administração e Planejamento em Saúde, Escola Nacional de Saúde Pública Sergio Arouca,

Fundação Oswaldo Cruz. R. Leopoldo Bulhões 1480, Manguinhos. 21.041-210 Rio de Janeiro RJ Brasil. camillafcastro@gmail.com
Abstract Brazil has hosted mass gatherings (MG) and participants are exposed to health risks. The scope of this paper is to systematize the main relationships between $M G$ and the occurrence of disasters of interest to Public Health. Three methodological strategies were used: systematic search and review of the literature on $M G$; systematization of the main information on MG; and selection and systematization of the references located for the theme of disaster in the search for key words, title or abstract. 28 references for MG were selected, mostly scientific papers, literature reviews, publications in 2012 and sports events. The main subjects addressed were surveillance systems and planning of MG. There was little analytical information about $M G$, as well as the importance of non-communicable events for the occurrence of disasters with emphasis on those related to crowds. It is important to consider the risk of disasters when planning $M G$ to increase responsiveness. Strategic areas such as medical emergencies, surveillance of communicable diseases and vectors, food and health service security, environmental health and laboratories should be considered. The importance of analytical research and registration of experience acquired in $M G$ should be stressed for disaster risk reduction.

Key words Mass gatherings, Public health, Health risk, Disasters
Resumo O Brasil tem sediado eventos de massa (EM) e seus participantes estão sujeitos a riscos à saúde. Objetivou-se sistematizar as principais relações, de interesse da Saúde Pública, entre eventos de massa e ocorrência de desastres. Foram realizadas três estratégias metodológicas: revisão da literatura de EM, com busca sistemática, sistematização das principais informações sobre EM e seleção e sistematização das referências que tratavam do tema desastre em suas palavras-chave, título ou resumo. Foram selecionadas 28 referências sobre EM, a maioria artigo científico, revisão de literatura, de 2012 e de natureza esportiva. Os principais assuntos abordados foram sistemas de vigilância e planejamento dos EM. Evidenciou-se a pouca informação analítica sobre os EM, bem como a importância de eventos não-transmissíveis para a ocorrência de desastres, com destaque aos relacionados à multidão. $O$ risco de desastres deve ser considerado no planejamento dos EM, para aumentar a capacidade de resposta considerando áreas estratégicas como emergência médica, vigilância de doenças transmissíveis e vetores, segurança alimentar e dos serviços de saúde, saúde ambiental e laboratório. Ressalta-se a importância de pesquisas analíticas e de registro das experiências relacionadas aos EM, para a redução de risco de desastres.

Palavras-chave Eventos de massa, Saúde pública, Risco sanitário, Desastres 


\section{Introdução}

Eventos de Massa são reuniões de grande contingente de pessoas, em geral motivados por atividades laborais, políticas, esportivas, religiosas ou lúdicas, que ocorre de forma pré-programada ou não, e que, em geral, acarretam consequências em diversos setores da sociedade, inclusive na Saúde Pública ${ }^{1,2}$.

A concentração de pessoas de origem nacional e internacional, de hábitos e culturas diferentes, expostas a distintos fatores de risco à saúde e portadoras de enfermidades - que podem ser potencializadas e disseminadas -, constitui-se fator de risco à saúde e de danos ambientais, sendo necessários planejamento e interação adequados de diversos setores, públicos e privados, na prevenção, resposta e recuperação às possíveis emergências e desastres ${ }^{3,4}$.

Além de doenças transmissíveis, os participantes de eventos de massa estão sujeitos a infecções alimentares, aumento do consumo de drogas lícitas e ilícitas, acidentes (como incêndios, desmoronamentos, pisoteamentos e esmagamentos), doenças respiratórias (como asma), suicídios, picadas de animais e insetos, doenças relacionadas à temperatura e umidade (desidratação, insolação, queimaduras solares, afecções respiratórias e hipotermia), injúrias e lacerações, doenças cardiovasculares ${ }^{5,6}$. A Organização Mundial da Saúde (OMS) também enumera algumas ameaças à Saúde Pública, tais como doenças novas e emergentes, utilização de agentes químicos, biológicos, radiológicos ou nucleares (QBRN), conflitos, desastres naturais ou antropogênicos ${ }^{7}$. Nos grandes eventos, pode haver emergências relacionadas à saúde dos participantes, e a capacidade de resposta a elas é fundamental para que não haja ocorrência de desastres. Esta última se relaciona, não apenas à exposição de grande número de pessoas vulneráveis a perigos, que podem resultar em danos à saúde ${ }^{8}$, como também à baixa capacidade de recursos, da sociedade afetada, para lidar com a situação de emergência ${ }^{4,7}$.

Semelhante aos grandes eventos, a ocorrência de desastres exige capacidade de ação frente às possíveis emergências e necessidades de saúde da população acometida. Estudo publicado em 2012, sobre as enchentes (desastres naturais que ocorrem com grande frequência) e sua relação com a Saúde Pública, apresentou como consequências sobre a saúde diversas doenças, tais como diarreias e gastroenterites, cólera, febre tifoide, desnutrição, estados de estresse póstraumático, conjuntivites, infecções respiratórias agudas, dermatites e erupções cutâneas. Sobre prevenção e mitigação dos riscos e impactos desse tipo de desastre, o estudo aponta como ações a serem adotadas a capacidade de avaliar rapidamente as áreas afetadas, identificando impactos, riscos e necessidades sociais, ambientais e de saúde da população afetada, além da capacidade de resposta do setor saúde, que inclui a vigilância em saúde, controle de hospedeiros e vetores de doenças, vacinações, atenção e cuidado, educação e conscientização em relação aos riscos e problemas de saúde?.

Os setores públicos e privados devem estar aptos para sediar os grandes eventos satisfatoriamente e, para tal, devem seguir as recomendações da OMS de fortalecimento dos sistemas de gestão de riscos de desastres e redução dos mesmos nos três níveis de gestão governamental (municipal, estadual e nacional), e as de desenvolvimento das capacidades e aumento da resiliência dos sistemas de saúde ao lidar com múltiplas vítimas ${ }^{10,11}$.

O Brasil tem sido destino de turistas em eventos de massa de diferentes naturezas, que fazem parte do calendário nacional, como carnaval, réveillon e festas típicas regionais. Além disso, uma série de eventos esportivos mundiais está programada até o ano de 2016. O país tem desenvolvido iniciativas que vão desde a estruturação de grupos de trabalho, regulamentações nacionais e locais, até articulações inter e intrassetoriais, visando avaliação dos riscos, preparação e eventual resposta aos acidentes que possam ocorrer durante os eventos de grande porte.

Este trabalho objetivou sistematizar as principais relações de interesse da Saúde Pública, entre a realização de eventos de massa e a ocorrência de desastres, identificadas em pesquisa de maior porte, que buscou caracterizar as ações de Saúde Pública nos eventos de massa ${ }^{12}$. Desta maneira, acredita-se contribuir para o planejamento e gestão de grandes eventos, minimizando a possibilidade de ocorrência de desastres a eles relacionados.

\section{Materiais e métodos}

A primeira estratégia metodológica se apoiou na revisão de literatura, com busca sistemática, realizada nos meses de janeiro e fevereiro de 2013. Utilizaram-se as bases de dados do Medical Literature Analysis and Retrieval System Online (Medline), da ScienceDirect, da Biblioteca Virtual em Saúde (BVS), do World Health Organization Library Information System (WHOLIS) e do World 
Health Organization Institutional Repository for Information Sharing (WHO IRIS). Os descritores mass gathering, mass gathering medicine, $p u-$ blic health, community health, health surveillance, sanitary surveillance, event surveillance, health protection, health promotion foram combinados, na sequência e com operadores booleanos, considerando as características de cada base de dados. Os critérios de busca adotados foram o período de publicação (de 2000 a 2012), o idioma (português, inglês e espanhol) e o acesso público eletrônico. As publicações foram selecionadas a partir da leitura de título e resumo, sendo mantidas aquelas que se referiam a Saúde Pública e eventos de massa.

Sistematizaram-se, num primeiro momento, as informações referentes à literatura selecionada (nome do(s) autor(es), ano de publicação, periódico, país/região do estudo (local de referência do estudo), tipo de publicação (artigo científico, comunicação rápida, documento oficial, editorial, estudo de opinião, livro, relatório) e assuntos abordados.

O tipo de publicação reflete as definições assumidas nas respectivas referências selecionadas. Os assuntos foram classificados em 18 categorias analíticas, com base na OMS, que foram: laboratórios; controle e prevenção de infecções; serviços médicos de emergência e hospitalares; saúde do viajante (incluindo a capacidade de tratamento); portos, aeroportos e fronteiras; promoção da saúde (condutas saudável, incentivo a atividades físicas, sexo seguro, redução do consumo álcool e tabaco, entre outros); agentes químicos, biológicos, radiológicos ou nucleares; acidentes com múltiplas vítimas, desastres, terrorismo e bioterrorismo; alimentos e segurança alimentar; controle da água; controle de vetores; saúde ambiental; bens e produtos para saúde (excetuando-se alimentos); controle de multidão; informação, comunicação e sistemas de alerta; sistemas de vigilância; planejamento e gestão; e legado à saúde ${ }^{13}$.

A segunda estratégia metodológica baseou-se na leitura integral dos textos selecionados e buscou sistematizar as principais informações sobre os eventos de massa: natureza do evento (esportivo, cultural, religioso e sociopolítico); local de realização do evento; situações de risco à saúde, potencial ou diretamente, relacionadas a eventos de massa.

$\mathrm{Na}$ terceira estratégia, as referências que tratavam do tema desastre em suas palavras-chave, título, resumo ou de forma expressiva no texto, foram selecionadas e sistematizadas. A associação entre o tema eventos de massa e desastres, que abordaram aspectos do planejamento e da gestão, foi considerada relação indireta. A associação propriamente dita entre eventos de massa, que resultaram em desastres, foi considerada relação direta.

\section{Resultados}

\section{Eventos de massa e Saúde Pública}

A busca bibliográfica recuperou 297 referências, das quais 28 foram selecionadas. $\mathrm{O}$ maior número de publicações selecionadas (28,6\%) pertencia ao periódico BMC Public Health. O ano de 2012 concentrou 32\% (9) das publicações; os países/regiões de estudo mais abordados foram Grécia e Reino Unido (17,8\% cada), e Austrália (14,3\%) (Quadro 1). Dezenove publicações $(67,8 \%)$ eram artigos científicos e a abordagem utilizada, pela maior parte das referências selecionadas, foi a de estudos de revisão da literatura (25\%). Apenas uma publicação abordou, de forma mais analítica, mudanças efetuadas no sistema de monitoramento de doenças infecciosas ${ }^{14}$ (Quadro 1).

No total das referências selecionadas, a principal natureza dos eventos foi a esportiva (68\%), seguida pelas referências que abordavam diferentes naturezas de eventos (18\%) (Quadro 2).

Entre os assuntos abordados nas referências selecionadas, destacaram-se os temas sobre: sistemas de vigilância (17,5\%), planejamento e gestão (12,4\%); alimentos e segurança alimentar (9,3\%); promoção da saúde $(8,2 \%)$; controle de água (8,2\%); saúde ambiental (8,2\%); controle e prevenção de infecções $(7,2 \%)$; serviços médicos de emergência e hospitalares $(6,2 \%)$; e acidentes com múltiplas vítimas, desastres, terrorismo e bioterrorismo (6,2\%). Ressalta-se que $71,4 \%$ das publicações abordaram mais de um assunto. O Quadro 2 demonstra também que, das 28 publicações selecionadas para o estudo, $21,4 \%$ (6) relacionaram, de forma direta ou indireta, a realização de grandes eventos com a ocorrência de desastres.

\section{Eventos de massa e desastres}

As quatro publicações que relacionaram de forma indireta eventos de massa e desastres abordaram especialmente os aspectos de planejamento, gestão, informação e capacidade de resposta às emergências ${ }^{13,15-17}$. Nas duas publicações em 
Quadro 1. Descrição dos autores, ano de publicação, país/região do estudo e tipo de publicação. Período de 2000 a 2012 .

\begin{tabular}{|c|c|c|c|c|}
\hline $\begin{array}{c}N^{\circ} \text { da Referência } \\
\text { Bibliográfica }\end{array}$ & Autor(es) & $\begin{array}{c}\text { Ano de } \\
\text { Publicação }\end{array}$ & $\begin{array}{l}\text { País/Região } \\
\text { do Estudo }\end{array}$ & Tipo de Publicação \\
\hline 1 & Fapore et al. ${ }^{18}$ & 2000 & EUA & Relatório \\
\hline 2 & Hanslik et al. ${ }^{19}$ & 2001 & França & Artigo Científico \\
\hline 3 & Jorm $^{20}$ & 2003 & Austrália & Artigo Científico \\
\hline 4 & Hadjichristodoulou et al. ${ }^{21}$ & 2005 & Grécia & Artigo Científico \\
\hline 5 & Muscatello et al. ${ }^{22}$ & 2005 & Austrália & Artigo Científico \\
\hline 6 & Coletta et al. ${ }^{23}$ & 2006 & EUA & Relatório \\
\hline 7 & Soteriades et al. ${ }^{24}$ & 2006 & Grécia & Artigo Científico \\
\hline 8 & Hadjichristodoulou et al. ${ }^{25}$ & 2006 & Grécia & Artigo Científico \\
\hline 9 & Tsouro e Efstathiou ${ }^{13}$ & 2007 & Grécia & Livro (Guias e Diretrizes) \\
\hline 10 & Yancey II et al. ${ }^{15}$ & 2008 & África do Sul & Artigo Científico \\
\hline 11 & Enock e Jacobs ${ }^{26}$ & 2008 & $\mathrm{NE}$ & Artigo Científico \\
\hline 12 & Marano e Freedman ${ }^{27}$ & 2008 & $\mathrm{NE}$ & Estudo de opinião \\
\hline 13 & Thackway et al. ${ }^{2}$ & 2009 & Austrália & Estudo de opinião \\
\hline 14 & Badekas et al..$^{28}$ & 2009 & Grécia & Artigo Científico \\
\hline 15 & Loncarevic et al. ${ }^{29}$ & 2009 & Sérvia & Comunicação Rápida \\
\hline 16 & McCartney et al. ${ }^{30}$ & 2010 & NE & Artigo Científico \\
\hline 17 & McCartney et al. ${ }^{31}$ & 2010 & Reino Unido & Artigo Científico \\
\hline 18 & Lund et al. ${ }^{16}$ & 2011 & Canadá & Artigo Científico \\
\hline 19 & $\mathrm{WHO}^{17}$ & 2011 & $\mathrm{NE}$ & Documento Oficial \\
\hline 20 & Polkinghorne et al. ${ }^{32}$ & 2012 & Austrália & Artigo Científico \\
\hline 21 & Takla et al. ${ }^{14}$ & 2012 & Alemanha & Artigo Científico \\
\hline 22 & Tew et al. ${ }^{33}$ & 2012 & Reino Unido & Editorial \\
\hline 23 & Carmont $^{34}$ & 2012 & Reino Unido & Editorial \\
\hline 24 & Severi et al. ${ }^{35}$ & 2012 & Reino Unido & Artigo Científico \\
\hline 25 & Heinsbroek et al. ${ }^{36}$ & 2012 & Reino Unido & Artigo Científico \\
\hline 26 & Soomaroo e Murray ${ }^{37}$ & 2012 & $\mathrm{NE}$ & Artigo Científico \\
\hline 27 & Tewari et al. ${ }^{38}$ & 2012 & Índia & Artigo Científico \\
\hline 28 & Soomaroo e Murray ${ }^{39}$ & 2012 & $\mathrm{NE}$ & Artigo Científico \\
\hline
\end{tabular}

NE = Não Especificado

que a relação era direta, elencaram-se as lições aprendidas com a ocorrência de desastres durante os eventos de massa ${ }^{37,39}$.

Em 2009, na Itália, o Fórum Global sobre eventos de massa teve como objetivo a revisão dos recursos e ferramentas necessárias para a realização de eventos de massa por especialistas e organizadores de diferentes eventos. O compartilhamento de informações relevantes sobre eventos de massa configurou-se uma importante estratégia, uma vez que subsidiaria o planejamento de futuros eventos, aumentando a capacidade de organização e a segurança sanitária ${ }^{17}$. Além disso, considerou que a gestão de desastre deveria 
Quadro 2. Evento de massa, natureza, assuntos abordados e relação com desastres. Período de 2000 a 2012.

\begin{tabular}{|c|c|c|c|c|}
\hline $\begin{array}{c}\mathrm{N}^{\circ} \text { da Referência } \\
\text { Bibliográfica }\end{array}$ & Eventos de Massa Abordados & $\begin{array}{c}\text { Natureza } \\
\text { dos eventos }\end{array}$ & $\begin{array}{l}\text { Assuntos } \\
\text { abordados }\end{array}$ & $\begin{array}{l}\text { Relação } \\
\text { com } \\
\text { desastres }\end{array}$ \\
\hline 1 & $\begin{array}{l}\text { Rainbow Family of Living Light Annual } \\
\text { Gathering, EUA (1999) }\end{array}$ & Cultural & $3 ; 5 ; 17$ & Não \\
\hline 2 & Copa do Mundo FIFA da França (1998) & Esportivo & 18 & Não \\
\hline 3 & $\begin{array}{l}\text { Olimpíadas e Paraolimpíadas de Sydney (2000); } \\
\text { Sydney Gay and Lesbian Mardi Gras (2000); } \\
\text { Ano Novo, Austrália (1999-2000) } \\
\text { Olimpíadas e Paraolimpíadas de Los Angeles } \\
\text { (1984), de Barcelona (1992) e de Atlanta (1996) }\end{array}$ & Esportivo & $\begin{array}{c}3 ; 5 ; 8 ; 12 ; 15 ; \\
18\end{array}$ & Não \\
\hline 4 & Olimpíadas e Paraolimpíadas de Atenas (2004) & Esportivo & $3 ; 5 ; 15 ; 18$ & Não \\
\hline 5 & Copa do Mundo de Rugby, Austrália (2003) & Esportivo & 18 & Não \\
\hline 6 & $\begin{array}{l}\text { Acampamento de jovens por } 10 \text { dias em } \\
\text { Virgínia, EUA (Julho, 2005) }\end{array}$ & Cultural & $8 ; 18$ & Não \\
\hline 7 & Olimpíadas e Paraolimpíadas de Atenas (2004) & Esportivo & 14 & Não \\
\hline 8 & Olimpíadas e Paraolimpíadas de Atenas (2004) & Esportivo & $3 ; 5 ; 15 ; 18$ & Não \\
\hline 9 & Olimpíada e Paraolimpíada de Atenas (2004) & Esportivo & $\begin{array}{l}1 ; 2 ; 3 ; 4 ; 5 ; 6 \\
7 ; 8 ; 9 ; 10 ; 12 \\
13 ; 14 ; 15 ; 16 \\
\quad 17 ; 18\end{array}$ & Indireta \\
\hline 10 & Copa do Mundo FIFA da África do Sul (2010) & Esportivo & $\begin{array}{l}1 ; 3 ; 11 ; 12 \\
\quad 17 ; 18\end{array}$ & Indireta \\
\hline 11 & $\begin{array}{l}\text { Olimpíadas e Paraolimpíadas de Atlanta (1996), } \\
\text { de Sydney (2000) e de Londres (2012) }\end{array}$ & Esportivo & $\begin{array}{c}3 ; 5 ; 8 ; 9 ; 12 \\
14 ; 15 ; 18\end{array}$ & Não \\
\hline 12 & Olimpíadas e Paraolimpíadas de Pequim (2008) & Esportivo & $8 ; 16 ; 18$ & Não \\
\hline 13 & $\begin{array}{l}\text { Olimpíadas e Paraolimpíadas de Sydney (2000); } \\
\text { Copa do Mundo de Rugby, Austrália (2003); } \\
\text { Fórum da Cooperação Econômica da Ásia e do } \\
\text { Pacífico (APEC), Austrália (2007); Dia Mundial } \\
\text { da Juventude, Austrália (2008) }\end{array}$ & Esportivo & $3 ; 12 ; 14 ; 15 ; 18$ & Não \\
\hline 14 & Olimpíadas e Paraolimpíadas de Atenas (2004) & Esportivo & $17 ; 18$ & Não \\
\hline 15 & $\begin{array}{l}\text { XXV Universitária de Verão em Belgrado, } \\
\text { Sérvia (2009); X Festival de Música EXIT em } \\
\text { Voivodina, Sérvia (2009) }\end{array}$ & $\begin{array}{l}\text { Diferentes } \\
\text { naturezas } \\
\text { de evento }\end{array}$ & $8 ; 12 ; 16 ; 18$ & Não \\
\hline 16 & $\begin{array}{l}\text { Olimpíadas e Paraolimpíadas de Londres } \\
\text { (2012); Jogos Commonwealth, de Glasgow, } \\
\text { Escócia (2014); Grandes Eventos Esportivos } \\
\text { (entre } 1978 \text { e 2008) }\end{array}$ & Esportivo & 11 & Não \\
\hline
\end{tabular}




\begin{tabular}{|c|c|c|c|c|}
\hline \multicolumn{5}{|c|}{ Quadro 2. continuação } \\
\hline $\begin{array}{c}\mathrm{N}^{\circ} \text { da Referência } \\
\text { Bibliográfica }\end{array}$ & Eventos de Massa Abordados & $\begin{array}{c}\text { Natureza } \\
\text { dos eventos }\end{array}$ & $\begin{array}{l}\text { Assuntos } \\
\text { abordados }\end{array}$ & $\begin{array}{c}\text { Relação } \\
\text { com } \\
\text { desastres }\end{array}$ \\
\hline 17 & $\begin{array}{l}\text { Olimpíadas e Paraolimpíadas de Londres } \\
\text { (2012) }\end{array}$ & Esportivo & $11 ; 12 ; 14 ; 15$ & Não \\
\hline 18 & $\begin{array}{l}\text { Olimpíada de Inverno em Vancouver, Canadá } \\
(2010) \text {; } \\
\text { Jogos Mundiais dos Policiais e Bombeiros, } \\
\text { Vancouver, Canadá (2009); } \\
\text { Copa do Mundo FIFA, campeonatos mundiais } \\
\text { de eventos esportivos, numerosas corridas, } \\
\text { festivais e concertos. }\end{array}$ & $\begin{array}{l}\text { Diferentes } \\
\text { naturezas } \\
\text { de evento }\end{array}$ & $1 ; 12 ; 17$ & Indireta \\
\hline 19 & $\begin{array}{l}\text { Expo } 2010 \text { Shanghai China (2010); Olimpíadas } \\
\text { e Paraolimpíadas do Rio de Janeiro (2016); } \\
\text { Hajj, Mecca, Arábia Saudita (2009); }\end{array}$ & $\begin{array}{l}\text { Diferentes } \\
\text { naturezas } \\
\text { de evento }\end{array}$ & $1 ; 12$ & Indireta \\
\hline 20 & $\begin{array}{l}\text { Festival de Música Country em Tamworth, } \\
\text { Austrália (2011) }\end{array}$ & Cultural & $\begin{array}{c}3 ; 5 ; 8 ; 12 ; 15 ; \\
18\end{array}$ & Não \\
\hline 21 & $\begin{array}{l}\text { Copa do Mundo FIFA da Alemanha (2006) e } \\
\text { Copa do Mundo FIFA Feminino, Alemanha } \\
\text { (2011) }\end{array}$ & Esportivo & 18 & Não \\
\hline 22 & $\begin{array}{l}\text { Olimpíadas e Paraolimpíadas de Londres } \\
\text { (2012) }\end{array}$ & Esportivo & $11 ; 14$ & Não \\
\hline 23 & $\begin{array}{l}\text { Olimpíadas e Paraolimpíadas de Londres } \\
\text { (2012) }\end{array}$ & Esportivo & $11 ; 14$ & Não \\
\hline 24 & $\begin{array}{l}\text { Olimpíadas e Paraolimpíadas de Londres } \\
\text { (2012) }\end{array}$ & Esportivo & 18 & Não \\
\hline 25 & $\begin{array}{l}\text { Olimpíadas e Paraolimpíadas de Londres } \\
(2012)\end{array}$ & Esportivo & 18 & Não \\
\hline 26 & $\begin{array}{l}21 \text { eventos em massa que terminaram em } \\
\text { desastres documentados ao redor do mundo }\end{array}$ & $\begin{array}{c}\text { Diferentes } \\
\text { naturezas } \\
\text { de eventos }\end{array}$ & $1 ; 6 ; 12 ; 17$ & Direta \\
\hline 27 & $\begin{array}{l}\text { Peregrinação ao Festival Religioso Hindu Magh } \\
\text { Mela no Norte da Índia (2011) }\end{array}$ & Religioso & 14 & Não \\
\hline 28 & $\begin{array}{l}22 \text { eventos em massa que terminaram em } \\
\text { desastres documentados ao redor do mundo }\end{array}$ & $\begin{array}{c}\text { Diferentes } \\
\text { naturezas } \\
\text { de eventos }\end{array}$ & $1 ; 5 ; 12$ & Direta \\
\hline
\end{tabular}

Obs: Classificação baseada em Tsouro e Efstathiou ${ }^{13}$. (1) Acidentes com múltiplas vítimas, Desastres, Terrorismo e Bioterrorismo; (2) Agentes Químicos, Biológicos, Radiológicos e Nucleares (QBRN); (3) Alimentos e Segurança Alimentar; (4) Bens e Produtos para Saúde; (5) Controle de Água; (6) Controle de Multidão; (7) Controle de Vetores; (8) Controle e Prevenção de Infecções; (9) Informação, Comunicação e Sistemas de Alerta; (10) Laboratórios; (11) Legado à Saúde; (12) Planejamento e Gestão; (13) Portos, Aeroportos e Fronteiras; (14) Promoção da Saúde; (15) Saúde Ambiental; (16) Saúde do Viajante; (17) Serviços Médicos de Emergência e Hospitalares e (18) Sistemas de Vigilância. 
compor o rol de elementos a serem considerados durante o planejamento de eventos de massa ${ }^{17}$.

As experiências e lições obtidas durante os Jogos Olímpicos de Atenas 2004 evidenciaram uma série de aspectos relevantes, inclusive a necessidade de realizar planejamento e gestão de desastres $^{13}$. Alguns temas considerados importantes foram os relativos a liderança, operações e comando unificado e cooperação internacional em Saúde Pública para grandes eventos e situações emergenciais ${ }^{13}$. No que tange à saúde propriamente dita, foi sugerida a composição de grupos de caráter consultivo, com especialistas da saúde, nos temas relativos a: prevenção de doenças, promoção da saúde, análise e gestão de riscos e segurança sanitária, vigilância e alerta, laboratório, serviços médicos, controle de infecções, segurança da água e alimentos, desastres e resposta a emergências ${ }^{13,17}$.

Algumas áreas foram consideradas estratégicas, tais como ${ }^{13}$ :

1. Preparação da capacidade hospitalar, de cuidados à saúde e de emergências médicas em acidentes com múltiplas vítimas;

2. Vigilância de doenças e resposta a surtos, incluindo a vigilância epidemiológica e a resposta às doenças transmissíveis,

3. Sistema de vigilância sindrômica, preparação e resposta da Saúde Pública a acidentes envolvendo agentes explosivos, biológicos, químicos e radiológicos e nucleares;

4. Saúde ambiental e segurança alimentar, incluindo temas sobre vigilância da saúde ambiental, qualidade da água, suporte laboratorial; e

5. Monitoramento e controle de mosquitos e vetores.

No enfrentamento dos desafios que se apresentam na realização de eventos de massa, além do planejamento, a avaliação dos riscos para a Saúde Pública foi considerada importante ${ }^{15,17}$. Yancey II et al. ${ }^{15}$ sugeriram a elaboração prévia de plano de preparação para desastres, e enfatizaram a importância da atuação de diferentes órgãos, que devem interagir na construção, debate e revisão de cada detalhe do plano, visando aumentar a capacidade de resposta do sistema médico-hospitalar frente a esse tipo de situação.

$\mathrm{Na}$ elaboração de planos de resposta a acidentes com múltiplas vítimas, as etapas de avaliação de necessidades e riscos podem elucidar perigos potenciais de áreas geográficas específicas, facilitando a preparação por parte das instituições interessadas, antecipando problemas, auxiliando o processo de priorização das políticas a serem adotadas e evitando preparações desnecessárias ${ }^{13}$.
No que tange às questões relacionadas aos desastres, os autores sugeriram definir os processos psicossociais demandados para apoio às vítimas, seus familiares e os profissionais envolvidos com as primeiras etapas de resposta. Sugeriram também definir plano de apoio mútuo e assistência internacional para casos que ultrapassem as capacidades de resposta locais ${ }^{13}$.

Os autores ressaltaram que o plano de preparação e resposta para acidentes com múltiplas vítimas deve estar em concordância com as legislações e regulamentações vigentes. Recomendaram estabelecer adequada cadeia de comando, controle e coordenação de ações, bem como possíveis transferências de responsabilidades entre comandos. Nas etapas pré-hospitalares, sugeriram que a autoridade responsável pelo comando geral seja definida de acordo ao tipo de acidente ocorrido. As ações pré-hospitalares, segundo os autores, devem seguir protocolos pré-determinados e incluem as etapas de descontaminação das vítimas, triagem, tratamento no local do acidente, transporte seguro das vítimas e a comunicação coordenada entre os responsáveis pelos serviços de atendimento médico-hospitalar, a autoridade em comando no local do desastre e o departamento nacional envolvido, garantindo que todas as necessidades sejam atendidas. É necessário assegurar a integridade dos corpos e a supervisão dos profissionais envolvidos ${ }^{13}$, incluindo o controle de acesso às áreas de guarda dos mortos ${ }^{13,15}$. Yancey II et al. ${ }^{15}$ sugeriram que os serviços de patologia forense, no caso de mortes em massa, sejam acionados, para prestar apoio à polícia e ajudar na identificação das vítimas.

O planejamento das etapas hospitalares, na resposta a acidentes com múltiplas vítimas, deve contemplar como componentes básicos a resposta integrada entre as agências envolvidas, a logística e a cadeia de suprimentos (de medicamentos, materiais médico-hospitalares, banco de sangue, equipamentos de proteção individual - EPI, detectores de radiação, entre outros), a segurança, os cuidados clínicos, os recursos humanos e as relações públicas ${ }^{13}$. Essas etapas incluem: a informação das capacidades dos sistemas hospitalares para a distribuição de pacientes; a designação de um centro de comando e controle no hospital; a adequada notificação do tipo de acidente ocorrido e suas demandas; os meios de comunicação interna e externa ao hospital; a segurança dos profissionais e equipamentos envolvidos; a proteção da infraestrutura hospitalar (contemplando os planos operacionais de resposta para contaminação do sistema de ventilação, interrupção 
do fornecimento de eletricidade, água, gases medicinais e outras funções críticas); a triagem, descontaminação e tratamento de pacientes; a operacionalização das ações de médicos especialistas, estudantes e voluntários; o apoio psicossocial, mental e espiritual às vítimas, seus familiares e profissionais de saúde a curto e longo prazos e o treinamento de pessoal, incluindo todos os cenários e protocolos pré-definidos ${ }^{13}$.

Nos casos em que as necessidades demandadas ultrapassarem a capacidade de resposta de um único hospital, deve-se elaborar resposta regional coordenada, estabelecer uma base de cuidados para pacientes menos graves próxima ao local do acidente, acionar cooperação nacional e internacional quando demandada, contemplando o transporte de pacientes e um plano de comunicação em massa ${ }^{13}$.

Lund et al. ${ }^{16}$ consideram relevante a experiência adquirida por cidades que sediaram eventos de massa, mesmo quando surpreendidas pela ocorrência de desastres. A disponibilidade de informações que visam contribuir para prevenção de riscos relacionados à concentração de grande número de pessoas subsidia a melhor capacidade de preparação e resposta a desastres e ataques terroristas. Quanto maior for a experiência no planejamento, preparação e prestação de serviços relacionados com a saúde em eventos de massa, mais os profissionais de saúde tendem a fortalecer sua prática na prestação de cuidados médicos.

Os autores elencam questões que são apontadas como essenciais para o sucesso das ações médicas em grandes eventos, tais como: a natureza do evento; o planejamento de ações pré e durante o evento; a organização da equipe de profissionais de saúde e seu processo de trabalho; a capacidade de resposta do sistema de saúde, inserindo-se aqui os serviços de emergência estruturados no local do evento e fora dele. Eles também postulam que os eventos de massa e os desastres (sejam eles de origem natural ou antropogênica) possuem muitos pontos em comum, como por exemplo: os meios de comunicação que utilizam, os limitados suportes e equipamentos médicos disponíveis, a necessidade de triagem para os atendimentos médicos, a ação coordenada de departamentos e agências de logística e operações, transportes, atendimento médico de emergência, entre outras semelhanças.

Estes autores elencam as similaridades e diferenças entre o que denominam Medicina dos Eventos de Massa (MEM) e Medicina de Desastres $(\mathrm{MD})^{16}$. No que diz respeito à logística, as similaridades são pela localização, geralmente temporária das estruturas, inclusive sanitárias; variabilidade de aspectos relativos à temperatura e umidade; necessidade de água potável; limitação orçamentária, de energia elétrica e de equipamentos médicos; desafios para a comunicação; e necessidade de ação coordenada na resposta dos diferentes órgãos. Quanto aos recursos humanos, ambas contam com equipe interdisciplinar remunerada ou não, trabalhando em ambiente não familiar e com participação de voluntários, não remunerados. Há variação na gravidade dos casos a serem atendidos, o que faz da triagem uma prática importante. Finalmente, no que tange à previsibilidade das necessidades, o planejamento da MEM e da MD baseia-se na experiência pregressa e na literatura e o tempo dos eventos é, em geral, limitado de algumas horas a poucos dias.

Algumas diferenças, entretanto, podem ocorrer: a resposta aos desastres pode demandar atendimento hospitalar a maior número de pessoas afetadas. O diagnóstico e a definição da responsabilidade do tratamento, por vezes, só podem ser feitos no local dos desastres, e alguns membros passam a integrar a equipe pela proximidade em que se encontram e de acordo com a demanda, e não por escolha, uma vez que é mais difícil prever as necessidades. Em MD, equipes de liderança no local são de extrema importância e pode tornarse necessário o suporte militar. Os autores ressaltam que a Medicina dos Eventos de Massa encontra-se embrionária e a literatura existente ainda é muito descritiva, havendo necessidade de acesso às informações mais analíticas sobre experiências similares cuja disponibilidade é escassa ${ }^{16}$.

Há necessidade de integração entre a Saúde Pública e a emergência médica, quando da elaboração do plano e definição dos recursos necessários à realização dos eventos de massa. Para evitar desastres e limitar as conseqüências de eventuais acidentes, os autores lembram alguns princípios de prevenção, dentre eles: o controle da densidade de espectadores com a ativação de um painel público orientando a dispersão, em caso de ultrapassar o limiar previsto; a separação entre o público e o campo com material projetado para absorver a pressão da multidão, de forma a evitar esmagamentos e asfixias em caso de competições esportivas; a acessibilidade de todas as especialidades médicas para o atendimento imediato dos feridos; triagem extraordinária e treinamento prévio de todos os profissionais da emergência médica ${ }^{15}$.

O plano de preparação e resposta a desastres deve ter conteúdo que oriente como será realizado o atendimento de emergência, com que 
recursos e em que prazos os recursos serão mobilizados e disponibilizados. Não basta apenas a elaboração do plano. Os gestores e profissionais que atuam na gestão, segurança, assistência médica no local do evento e nos hospitais, organização e realização do evento devem poder conhecer e opinar sobre seu conteúdo. A revisão do plano pela equipe de emergência é um importante elemento. Outros elementos são: a elaboração de planos de emergência dos hospitais; acesso seguro de ambulâncias no local do evento e resposta a acidentes adequadamente tratada com os profissionais. Os autores orientaram ainda para a definição de papéis alternativos para quem atua na emergência médica, de forma que cada profissional saiba sua função: triagem, atendimento de urgência de acordo com a gravidade, atendimento no local do desastre ou no preparo e transporte de ambulâncias ${ }^{15}$.

Recentes estudos, oriundos de revisão sistemática da literatura, buscaram analisar desastres ocorridos durante a realização de eventos de massa, documentando as lições que podem auxiliar na preparação de futuros eventos. Em ambas as revisões, os autores concluíram que os grandes eventos possuem enorme potencial de tensionar o sistema local de saúde.

Uma revisão dedicou-se a analisar, exclusivamente, os desastres relacionados à temperatura e ao ambiente, de 1988 a 2011. Os autores categorizaram os achados em doenças resultantes de frio e calor, tempestades e raios, e ocorrência de surtos $^{37}$. Indicaram que a desidratação e as doenças relacionadas ao calor são ocorrências comuns, sendo interessante fazer a recomendação aos participantes de que bebam líquido, procurem a sombra e saibam reconhecer os sintomas de desidratação ${ }^{37}$. Em ambientes frios, problemas de saúde importantes são os respiratórios e a hipotermia. Em locais onde pode haver tempestade de neve há relatos de desastres devido ao desabamento de telhado e estruturas físicas sobre os participantes. Os autores adaptaram o modelo preditivo de Hartman et al. ${ }^{40}$, que se utiliza de alguns parâmetros (tempo, número de participantes, consumo esperado de álcool, perfil do evento em termos de calmo a animado), para classificá-los em de maior, intermediário ou menor consumo de recursos. No que tange às questões relacionadas aos raios, foram relatados dois acidentes, a lesão de uma pessoa falando ao telefone celular, em estádio, e a necessidade de evacuação durante um jogo escolar. Os autores sugeriram que, na possibilidade de ocorrência de raios e tempestades, seja feita avaliação do local, que estejam disponíveis sistemas de alerta precoce sobre o tempo e abrigos definidos, bem como protocolo para evacuação do local, sendo importante constar na programação e em folhetos as orientações sobre a evacuação e a segurança do evento. Os problemas relacionados à água contaminada referiram-se a casos de hepatite A por água de fonte, de surto de legionelose após uso de piscina, de leptospirose por água de lago, de shigelose por consumo de tofu mal cozido, e de E. Coli O157, resultante de contato com a pecuária local. Em Meca, foi relatado surto de cerca de 90 casos de doença meningocócica, com 14 mortes, o que resultou na recomendação, aos peregrinos, da vacinação antes de viagem à Arábia Saudita ${ }^{37}$.

Uma segunda revisão direcionou-se à análise de desastres relacionados à multidão. Os autores identificaram 156 artigos, a maioria descritiva, e apenas 21 deles possuíam informação suficiente para a análise das lições aprendidas em eventos de massa de diferentes naturezas, identificados entre 1971 e 2011. O maior número de vítimas letais foi por esmagamento em eventos religiosos (1.426 em 1990 e 346 em 2010) em Meca, na Arábia Saudita, e em evento aquático, no Camboja, em 2010, onde morreram 347 pessoas. $\mathrm{Na}$ Índia, 441 mortes ocorreram, em 1995, durante um casamento, após curto circuito em local com insuficiente segurança contra incêndio ${ }^{39}$.

Visando reduzir a morbidade e a mortalidade em eventos de massa, os autores sugeriram que o planejamento dos eventos de massa deve envolver os hospitais locais no que diz respeito à preparação dos serviços médicos. Eles devem fazer seu planejamento específico em caso de acidentes com múltiplas vítimas, incluir um centro hospitalar de comando, prever a forma de contato e a organização dos profissionais de saúde, bem como prover o envolvimento da rede hospitalar de maneira tempestiva ${ }^{39}$.

$\mathrm{Na}$ resposta às emergências médicas, aspectos importantes foram elencados, como o tempo de resposta, a comunicação entre os organizadores e os serviços de emergência, bem como a triagem dos casos no local. Neste sentido, os autores apontaram que o plano de emergência deve prever o treinamento em Medicina de Desastres da equipe no local do evento, de forma a poderem utilizar os métodos de triagem; sugeriram a análise do cuidado médico em grandes eventos, proposta por Sanders et al. ${ }^{41}$; e oferta de cuidados básicos em quatro minutos, suporte avançado de vida em oito minutos e transferência para um centro médico em 30 minutos $^{39}$. De acordo com as propostas de Hartman et $\mathrm{al}^{42}$, os autores destacaram a 
necessidade de recursos médicos para estádios de futebol durante a realização de eventos de massa e propõem: um profissional para primeiros socorros a cada 1.000 participantes; provisão de salas de atendimento de pronto-socorro no local, em que o número e os equipamentos necessários sejam definidos pela autoridade local; um médico treinado para prestar socorro avançado no local do evento, se for ultrapassado o total de 2.000 pessoas; no mínimo uma ambulância totalmente equipada, se a multidão esperada for maior que 5.000 participantes; e um veículo equipado com 50 macas, cobertores e equipamentos médicos, em caso de uma população participante maior que 25.000 pessoas.

\section{Discussão}

Os eventos de massa distinguem-se pelas condições climáticas, duração do evento, idade e comportamento da multidão, perigos específicos, uso de álcool e drogas, origem e número de participantes, distribuição espacial e atenção dos serviços públicos dispensada aos mesmos. Tamanha variabilidade acarreta diferentes escalas de preparação de sistemas de Saúde Pública e prestação de serviços médicos ${ }^{14,16}$. Este estudo identificou, nas referências selecionadas, um maior número de eventos de natureza esportiva (68\%), o que limita as informações necessárias sobre os demais tipos de eventos.

O predomínio de estudos de revisão da literatura, em geral voltados aos aspectos relacionados ao planejamento e gestão dos grandes eventos, encontrado neste estudo, vai, portanto, ao encontro da preocupação com a etapa de preparação dos eventos de massa. Alguns fatores, considerados cruciais para o sucesso de eventos de massa foram observados: a pré-identificação de fatores críticos; a avaliação, mitigação e gestão de riscos; os estudos de avaliação de impacto; o detalhamento dos planos de contingência; e o teste completo prévio de todos os planos estipu$\operatorname{lados}^{26}$. A literatura evidencia a importância do planejamento e gestão, com elaboração de Planos de Ação, que inclua a atuação em caso de acidentes e emergências médicas, com destaque, dentre outros, para: centros de comunicação e comando em Saúde Pública; sistemas de vigilância, segurança e saúde ambiental; surtos de doenças infecciosas e implicações de condições climáticas ${ }^{13}$.

Ressalte-se que a pouca literatura analítica encontrada sobre os grandes eventos é preocupação de alguns autores, que enfatizam a neces- sidade de se planejar considerando a experiência acumulada em eventos anteriores ${ }^{37,39}$. Zielinski ${ }^{43}$ corrobora essa visão quando afirma que a maioria dos estudos a respeito de doenças infecciosas em eventos de massa constitui-se em artigos relacionados à preparação dos eventos ou às análises teóricas, em contraste com uma minoria que provê informações à respeito de eventos ocorridos ou casos relatados.

As revisões sobre desastres ocorridos em eventos de massa ${ }^{37,39}$, ainda que tenham identificado uma centena de referências, também se depararam com a dificuldade de obtenção de informações mais completas sobre os eventos/desastres, o que fez com que a análise se restringisse a cerca de vinte eventos de massa.

Em geral, os eventos de massa são avaliados segundo suas características constitutivas, porém muitas vezes negligencia-se a discussão sobre sua potencialidade catastrófica ${ }^{30}$. Na literatura, estudo afirma que grandes eventos internacionais promovem uma maior conscientização e interesse por aspectos relacionados à capacidade de resposta às emergências, como forma de subsidiar a preparação e a resposta aos desastres, especialmente no que denominam de medicina de eventos de massa e medicina de desastres ${ }^{30}$.

Diversas situações podem acarretar agravos e mortes a um grande número de pessoas dentro de um mesmo espaço de tempo. De acordo com um estudo de revisão bibliográfica que teve como foco o evento bioterrorismo, essa questão tem sido objeto crescente de atenção da comunidade científica, de governantes e de militares, especialmente por sua letalidade indiscriminada $\mathrm{e}$ pela falta de controle sobre os agentes biológicos disseminados. Um elevado número de vítimas pode sobrecarregar os sistemas de saúde e gerar necessidade de aumentar o quantitativo de profissionais qualificados para essas situações, além da exigência de maior número de medicamentos, vacinas, materiais, equipamentos, informações e treinamentos apropriados ${ }^{44}$. Situações como esta indicam cenários de insegurança para populações e governantes e demandam capacidade de resposta adequada para atender essas possíveis e inesperadas ocorrências.

Sun et al. ${ }^{45}$ relataram sobre o risco de desastres como elemento chave na gestão de eventos de massa. Relacionam este tipo de evento a impactos na Saúde Pública e na política, enfatizando o potencial para ataques terroristas durante a realização de Jogos Olímpicos. A relação eventos de massa, desastres e ataques terroristas é corroborada por outros autores ${ }^{1,24}$. 
A opinião de que as experiencias nos grandes eventos podem orientar (e devem contemplar) a atuação em desastres envolvendo grande contingente de pessoas, em contextos diferenciados como o de doenças infecciosas e de situações climáticas adversas, é compartilhada pela Organização Mundial de Saúde ${ }^{1,17}$.

Outros fatores de risco descritos, nas revisões analisadas neste estudo ${ }^{37,39}$, como facilitadores para a ocorrência de desastres, como alta concentração de pessoas, baixo controle de multidão, insuficiente segurança contra incêndios, estrutura de atendimento médico local, comunicação entre organização e equipe médica, condições de temperatura adversas, insuficiência de saídas de emergência e pontos de acesso ao evento, encontram ressonância na literatura ${ }^{1,46}$.

Este estudo pode identificar a importância de unidade de coordenação de saúde pública e de serviços médicos presentes interna e externamente no local do evento, que vai ao encontro a Sun et $a l .{ }^{45}$, quando elenca aspectos que demandam coordenação, como a avaliação e comunicação do risco, a assistência médica, a vigilância de doenças e de vetores, a resposta laboratorial, as imunizações, a educação em saúde, e a segurança dos alimentos e da água.

No Brasil, a Lei n. 10.671, de 15 de maio de $2003^{47}$, que dispõe sobre o Estatuto de Defesa do Torcedor, durante eventos esportivos, prevê que é dever do organizador disponibilizar um médico e dois enfermeiros para cada dez mil torcedores e disponibilizar uma ambulância para cada dez mil torcedores, recomendação aquém da sugerida por Hartman et al. ${ }^{42}$. Os organizadores respondem solidariamente quando houver prejuízos ao torcedor, decorrentes de falhas de segurança nos estádios. Pela norma brasileira, é direito do torcedor a higiene e qualidade das instalações físicas e dos produtos alimentícios vendidos no local do evento, sendo os últimos verificados pela vigilância sanitária.

Foi possível destacar, em consonância com Steffen et al.$^{48}$, que há importantes riscos à saúde, distintos da transmissão de doenças, cuja mortalidade é maior, como é o caso da morte por esmagamentos, especialmente quando não há controle da multidão, hiperdensidade de participantes, inadequada infraestrutura e planejamento. Estes autores consideram importante que haja mais pesquisas sobre os eventos de massa, os fatores de risco de agravos não transmissíveis e as boas práticas para mitigá-los. Além disso, ressaltam a falta de informação sobre eventos de grande porte espontâneos, como manifestações políticas e funerais, bem como os não-tradicionais, como em shoppings e aeroportos ${ }^{48}$. A National Disaster Management Authority, da Austrália, considera relevante o controle de multidão nos eventos de massa, a tal ponto que a ele dedicou uma atenção especial $^{49}$.

A OMS propõe estratégias para que o setor saúde possa construir sua capacidade de resposta a desastres. Uma das iniciativas consideradas chave é a de avaliação do risco e do perigo, com ênfase em dados retrospectivos de situações anteriores, que não se limitem a desastres, mas que contemplem acidentes ocorridos em eventos. Para estes autores, o Plano Nacional de Gestão de Eventos de Destruição em Massa inclui o estabelecimento de responsabilidades nacional, estaduais e municipais. Neste sentido, consideram importante a existência de planos para eventos de massa de diferentes naturezas (esportiva, religiosa, cultural), uma vez que a ocorrência de acidentes de grandes proporções pode exceder a capacidade de resposta local ${ }^{50}$. Alguns autores afirmam que muitas vidas poderiam ser salvas com um planejamento prévio efetivo, porém a limitada preparação e a pequena capacidade de reação da maioria das cidades ao redor do mundo acarretam grande prejuízo humano e material. Algumas cidades, após os desastres, não são capazes de fornecer suporte adequado para os sobreviventes ${ }^{8}$.

Diferentes estudos convergem para a importância da participação e cooperação entre as diferentes esferas de governo, a interdisciplinaridade, a intersetorialidade no planejamento e implementação de ações preventivas e de redução de agravos à saúde. Apontam como essencial o trabalho conjunto entre diversos setores da sociedade, com vista a melhor capacidade de resposta às situações que comprometam a vida e saúde de populações ${ }^{9,44}$.

No Brasil, uma das mais recentes normas, a Portaria GM/MS n ${ }^{\circ}$ 1.139/2013, destaca a necessidade de atuação coordenada entre os órgãos de Saúde Pública das três esferas governamentais, bem como o fornecimento de serviços de saúde especiais conforme avaliação de ameaças, vulnerabilidades e riscos à Saúde Pública ${ }^{51}$.

De acordo com o Decreto 7.616 de 2011, situações de desastres são consideradas como Emergência em Saúde Pública de Importância Nacional. A Força Nacional do Sistema Único de Saúde (FN-SUS) foi instituída pelo governo brasileiro e apresentada como programa que visa cooperar na execução de medidas de prevenção, assistência e repressão a situações epidemiológi- 
cas, de desastres ou de desassistência à população ${ }^{52}$. Em 2013 foi registrada a possibilidade de atuação da FN-SUS no auxílio às situações de desastres naturais provocados pela temporada de chuvas. A disponibilização de medicamentos, insumos e estrutura para atuar em situações de emergência foram citadas como ações previstas. A FN-SUS também atuou na "tragédia de Santa Maria”, grande incêndio ocorrido em evento de massa cultural, realizado em uma boate com ambiente fechado, que resultou na morte de 242 pessoas e deixou centenas de feridos ${ }^{53}$.

O governo brasileiro tem anunciado medidas adotadas para a melhoria da estrutura de saúde do país durante e após a realização do evento Copa do Mundo FIFA Brasil 2014. A Portaria GM/MS no 1.066, de maio de 2011 instituiu o Grupo de Trabalho de Preparação das Ações de Saúde para a Copa do Mundo FIFA 2014 - GT Copa, que visa estabelecer diretrizes, ações estratégicas e metas, bem como acompanhar a preparação das ações para a Copa do Mundo, realizando encontros com as 12 cidades-sede do mundial no Brasil. O planejamento das ações de saúde do GT-Copa relacionam-se a vigilância sanitária, a vigilância epidemiológica e a assistência à saúde e aos aspectos referentes à gestão e aos legados do evento ${ }^{54}$.

\section{Considerações Finais}

A concentração de estudos descritivos e de revisão bibliográfica traz informações mais gerais, dificultando a caracterização de outros eventos tão importantes como os de natureza esportiva. No Brasil, eventos culturais e religiosos têm sido realizados e estas experiências merecem maior atenção e registro, de forma a subsidiar estados e municípios a elaborarem seus respectivos planos de ação.

Os riscos à Saúde Pública durante a realização de grandes eventos devem ser contemplados nas etapas de planejamento e gestão das ações, especialmente no que diz respeito à gestão de emergências, evitando que os eventos danosos sejam de magnitude tal, que se transformem em desastres. A sistematização da produção bibliográfica publicada a respeito de eventos de massa relacionados à Saúde Pública revelou-se uma ferramenta interessante, ao fornecer subsídios científicos a esse planejamento, contribuindo para a realização bem sucedida de tais eventos.

Durante o estudo foi possível perceber a inter -relação entre os eventos de massa e os desastres, tanto em suas características constitutivas, como em suas necessidades de preparação. Sugere-se, inclusive, que as ações e medidas adotadas para a preparação de grandes eventos sejam estendidas ao contexto dos desastres a que estão suscetíveis. Em geral, o planejamento das ações exige esforço multidisciplinar e intersetorial, incluindo não apenas a área da Saúde Pública, mas também setores de logística, segurança, transporte e hotelaria.

A atuação frente aos riscos e à ocorrência de doenças transmissíveis têm sido uma das mais valorizadas e referidas na literatura. Aspectos relacionados à alta concentração de pessoas, às condições climáticas, como raios e tempestades e à temperatura, como calor e frio, devem ser considerados para minimizar a ocorrência de desastres durante a realização de eventos de massa. Algumas situações específicas foram observadas e merecem ser também contempladas. No que diz respeito à qualidade da água, além da verificação da central de abastecimento, dos reservatórios intermediários e da água fornecida para o consumo dos participantes, parece ser importante incluir a verificação daquela que será utilizada pelos frequentadores dos eventos de massa, assim como as de lagos, fontes e chafarizes próximos ao evento. As ações voltadas ao controle das multidões, durante a realização dos grandes eventos, são também de suma importância para a redução do risco de desastres.

Os recentes eventos com grande aglomeração de pessoas, como as manifestações populares, de caráter reivindicatório, ocorridas recentemente em todo Brasil, especialmente em capitais como São Paulo, Rio de Janeiro e Porto Alegre, indicam a necessidade de preparação urgente e elaboração de planos de ação com alta capacidade de resposta para as possíveis emergências em saúde pública oriundas dos mesmos, considerando situações de distintas naturezas e a possibilidade das ocorrências inesperadas.

Considerando a possibilidade de ocorrência de emergências em saúde pública e desastres durante a realização e após grandes eventos, é importante que a preparação para tais eventos inclua a elaboração de plano de ação que contemple a capacidade de resposta aos desastres. As medidas necessárias para a redução dos riscos sanitários em grandes eventos não se restringem a consciência do potencial para ocorrência de desastres e outras situações que colocam vidas humanas e o meio ambiente em perigo. Essas medidas demandam investimentos, inclusive financeiros, na organização dos serviços e na assistência à saúde. 


\section{Colaboradores}

CF Castro, DCM Simões, EV Delamarque e VL Edais Pepe participaram igualmente de todas as etapas de elaboração do artigo.

\section{Referências}

1. World Health Organization (WHO). Communicable disease alert and response for mass gatherings: Technical workshop. Genebra: WHO; 2008.

2. Thackway S, Churches T, Fizzell J, Muscatello D, Armstrong P. Should cities hosting mass gatherings invest in public health surveillance and planning? Reflections from a decade of mass gatherings in Sydney, Australia. BMC Public Health 2009; 8(9):324.

3. World Health Organization (WHO). Interim planning considerations for mass gatherings in the context of pandemic (H1N1) 2009 influenza. Genebra: WHO Press; 2009.

4. Naciones Unidas. Estratégia Internacional para la Reducción de Desastres de las Naciones Unidas: terminología sobre reducción del riesgo de desastres. Genebra: UNIRSD; 2009.

5. McCartney G, Thomas S, Thomson H, Scott J, Hamilton V, Hanlon P, Morrison DS, Bond L. The health and socioeconomic impacts of major multi-sport events: systematic review (1978-2008). BMJ 2010; 20(340):c2369.

6. Coletta M, Dewey L, White-Russell M, Powell T, Toney D, Cheek J, Wong D, Young P, Melius E, Sandhu S. Surveillance for early detection of disease outbreaks at an outdoor mass gathering - Virginia, 2005. MMWR Weekly 2006; 55(3):71-74.

7. World Health Organization (WHO). Strengthening health-system emergency preparedness: Toolkit for Assessing Health System Capacity for Crisis Management. Copenhague: WHO Regional Office for Europe; 2012.

8. Wisner B, Adams J. Environmental health in emergencies and disasters: a practical guide. Genebra: WHO Press; 2002.

9. Freitas CM, Ximenes EF. Enchentes e saúde pública: uma questão na literatura científica recente das causas, consequências e respostas para prevenção e mitigação. Cien Saude Colet 2012; 17(6):1601-1616.

10. World Health Organization (WHO). Strengthening national health emergency and disaster management capacities and resilience of health systems. Sixty-Fourth World Health Assembly. Geneva: WHO; 2011.

11. Freitas CM, Carvalho ML, Ximenes EF, Arraes EF, Gomes JO. Vulnerabilidade socioambiental, redução de riscos de desastres e construção da resiliência: lições do terremoto no Haiti e das chuvas fortes na Região Serrana, Brasil. Cien Saude Colet 2012; 17(6):1577-1586.

12. Castro CF. A vigilância sanitária nos eventos de massa internacionais: uma revisão de literatura [monografia]. Rio de Janeiro: Escola Nacional de Saúde Pública Sergio Arouca; 2013.
13. Tsouro AD, Efstathiou PA. Mass gatherings and public health: the experience of the Athens 2004 olympic games. Copenhague: WHO Press; 2007.

14. Takla A, Velasco E, Benzler J. The FIFA Women's World Cup in Germany 2011--a practical example for tailoring an event-specific enhanced infectious disease surveillance system. BMC Public Health 2012; 31(12):576-582.

15. Yancey AH 2nd, Fuhri PD, Pillay Y, Greenwald I. World Cup 2010 planning: an integration of public health and medical systems. Public Health 2008; 122(10):1020-1029.

16. Lund A, Gutman SJ, Turris SA. Mass gathering medicine: a practical means of enhancing disaster preparedness in Canada. CJEM 2011; 13(4):231-236.

17. World Health Organization (WHO). Global forum on mass gatherings: Rome, Italy, 26-29 October 2009. Geneva: WHO; 2011.

18. Fapore D, Lurie P, Moll M, Weltman A, Rankin J. Public Health Aspects of the Rainbow Family of Living Light Annual Gathering - Allegheny National Forest, Pennsylvania, 1999. MMWR Weekly 2000; 49(15):324-326.

19. Hanslik T, Boelle PY, Flahault A. Setting up a specific surveillance system of community health during mass gatherings. J Epidemiol Community Health 2001; 55(9):683-684.

20. Jorm LR. Watching the Games: public health surveillance for the Sydney 2000 Olympic Games. J Epidemiol Community Health 2003; 57(2):102-108.

21. Hadjichristodoulou C, Soteriades ES, Kolonia V, Falagas ME, Pantelopoulos E, Panagakos G, Mouchtouri V, Kremastinou J. Methodological aspects of a GIS-based environmental health inspection program used in the Athens 2004 Olympic and Para Olympic Games. BMC Public Health 2005; 2(5):93.

22. Muscatello DJ, Churches T, Kaldor J, Zheng W, Chiu C, Correll P, Jorm L. An automated, broad-based, near real-time public health surveillance system using presentations to hospital Emergency Departments in New South Wales, Australia. BMC Public Health 2005; 22(5):141-152.

23. Coletta M, Dewey L, White-Russell M, Powell T, Toney D, Cheek J, Wong D, Young P, Melius E, Sandhu S. Surveillance for Early Detection of Disease Outbreaks at an Outdoor Mass Gathering - Virginia, 2005. MMWR 2006; 55(3):71-74.

24. Soteriades ES, Hadjichristodoulou C, Kremastinou J, Chelvatzoglou FC, Minogiannis PS, Falagas ME. Health promotion programs related to the Athens 2004 Olympic and Para Olympic games. BMC Public Health 2006; 24(6):47-51. 
25. Hadjichristodoulou C, Mouchtouri V, Vaitsi V, Kapoula C, Vousoureli A, Kalivitis I, Chervoni J, Papastergiou P, Vasilogiannakopoulos A, Daniilidis VD, Kremastinou J. Management of environmental health issues for the 2004 Athens Olympic Games: is enhanced integrated environmental health surveillance needed in every day routine operation? BMC Public Health 2006; 6:306.

26. Enock KE, Jacobs J. The Olympic and Paralympic Games 2012: literature review of the logistical planning and operational challenges for public health. Public Health 2008; 122(11):1229-1238.

27. Marano C, Freedman DO. Global health surveillance and travelers' health. Curr Opin Infect Dis 2008; 22(5):423-429.

28. Badekas T, Papadakis SA, Vergados N, Galanakos SP, Siapkara A, Forgrave M, Romansky N, Mirones S, Trnka HJ, Delmi M. Foot and ankle injuries during the Athens 2004 Olympic Games. J Foot Ankle Res 2009; 2:9-16.

29. Loncarevic G, Payne L, Kon P, Petrovic V, Dimitrijevic D, Knezevic T, Medić S, Milic N, Nedelijković J, Seke K, Coulombier D. Public health preparedness for two mass gathering events in the context of pandemic influenza (H1N1) 2009--Serbia, July 2009. Euro Surveill 2009; 14(31):14-17.

30. McCartney G, Thomas S, Thomson H, Scott J, Hamilton V, Hanlon P, Morrison DS, Bond L. The health and socioeconomic impacts of major multi-sport events: systematic review (1978-2008). BMJ 2010; 20(340):c2369.

31. McCartney M, Ravelli RJ, Sinclair-Williams M. Health impact assessment of the 2012 London Olympic transport plans. Eur J Public Health 2010; 20(6):619-624.

32. Polkinghorne BG, Massey PD, Durrheim DN, Byrnes T, MacIntyre CR. Prevention and surveillance of public health risks during extended mass gatherings in rural areas: The experience of the Tamworth Country Music Festival, Australia. Public Health 2012; 127(1):32-38.

33. Tew GA, Copeland RJ, Till SH. Sport and exercise medicine and the Olympic health legacy. BMC Medicine 2012; 10:74.

34. Carmont M. Advances in sports nutrition, exercise and medicine: Olympic issues, the legacy and beyond. BMC Medicine 2012; 10:79.

35. Severi E, Heinsbroek E, Watson C, Catchpole M; HPA Olympics Surveillance Work Group. Infectious disease surveillance for the London 2012 Olympic and Paralympic Games. Euro Surveill 2012; 17(31):8-15.

36. Heinsbroek E, Said B, Kirkbride H; HPA USII Steering Group. A new surveillance system for undiagnosed serious infectious illness for the London 2012 Olympic and Paralympic Games. Euro Surveill 2012; 17(31):16-21.

37. Soomaroo L, Murray V. Weather and Environmental Hazards at Mass Gatherings. PLoS Curr 2012; 31(4):e4fca9ee30afc4.

38. Tewari S, Khan S, Hopkins N, Srinivasan N, Reicher S. Participation in mass gatherings can benefit well-being: longitudinal and control data from a North Indian hindu pilgrimage event. PLoS ONE 2012; 7(10):e47291.

39. Soomaroo L, Murray V. Disasters at Mass Gatherings: Lessons from History. PLoS Curr 2012; 31(1):1-10.

40. Hartman N, Williamson A, Sojka B, Alibertis K, Sidebottom M, Berry T, Hamm J, O'Connor RE, Brady WJ. Predicting resource utilisation at mass gatherings using a simplified stratification scoring model. Am J Emerg Med 2009; 27(3):337-343.
41. Sanders AB, Criss E, Steckl P. An analysis of medical care at mass gatherings. Ann Emerg Med 1986; 15(5):515-519.

42. Hartman N, Williamson A, Sojka Taylor LJ. Final Report into the Hillsborough Stadium Disaster, CM 962. London: HMSO; 1990.

43. Zielinski A. Enhanced surveillance at mass gatherings. Przegl Epidemiol 2009; 63(4):477-485.

44. Cardoso DR, Cardoso TAO. Bioterrorismo: dados de uma história recente de riscos e incertezas. Cien Saude Colet 2011; 16(Supl. 1):821-830.

45. Sun X, Keim M, He Y, Mahany M, Yuan Z. Reducing the risk of public health emergencies for the world's largest mass gathering: 2010 World Exposition, Shanghai China. Disaster Health 2013; 1(1):21-29.

46. Lombardo JS, Sniegoski CA, Loschen WA, Westercamp M, Wade M, Dearth S, Zhang G. Public Health Surveillance for Mass Gatherings. Johns Hopkins Apl Technical Digest 2008; 27(4):347-355.

47. Brasil. Lei n. 10.671, de 15 de maio de 2003. Dispõe sobre o Estatuto de Defesa do Torcedor e dá outras providências. Diário Oficial da União 2003; 16 maio.

48. Steffen R, Bouchama A, Johansson A, Dvorak J, Isla N, Smallwood C, Memish ZA. Non-communicable health risks during mass gatherings. Lancet Infect Dis 2012; 12(2):142-149.

49. National Disaster Management Authority. Position Paper on Crowd Management at places of Mass Gatherings, 2013. [acessado 2014 jan 29]. Disponível em: http:// ndma.gov.in/ndma/guidelines/crowdmanagement guideline.pdf

50. World Health Organization (WHO). Mass casualty management systems: strategies and guidelines for building health sector capacity. Genebra: WHO Press; 2007

51. Brasil. Portaria GM/MS n.1.139, de 10 de junho de 2013. Define, no âmbito do Sistema Único de Saúde (SUS), as responsabilidades das esferas de gestão e estabelece as Diretrizes Nacionais para Planejamento, Execução e Avaliação das Ações de Vigilância e Assistência à Saúde em Eventos de Massa. Diário Oficial da União 2013; 11 jun.

52. Brasil. Decreto n. 7.616, de 17 de novembro de 2011. Dispõe sobre a declaração de Emergência em Saúde Pública de Importância Nacional - ESPIN e institui a Força Nacional do Sistema Único de Saúde - FN-SUS. Diário Oficial da União 2011; 18 nov.

53. Valcarenghi A. Ministério da Saúde reforça Força Nacional do SUS para temporada de chuvas [internet]. Empresa Brasil de Comunicação - Agência Brasil. 2013 nov. 26 [acessado 2014 jun 14]. Disponível em: http:// ebcnare.de/1blTXxq

54. Conselho Nacional dos Secretários de Saúde (CONASS). Ações de Saúde para a Copa do Mundo FIFA 2014. 2012. [acessado 2014 fev 01]. Disponível em: http://www.conass.org.br/NOTAS\%20T\%C3\%89CNICAS\%202012/NT\%2004-2012\%20Сopa\%202014.pdf

Artigo apresentado em 23/03/2014

Aprovado em 05/06/2014

Versão final apresentada em 07/06/2014 


\section{ERRATA}

p. 3717

onde se lê:

Mass events, disasters and public health

leia-se:

Mass gatherings, disasters and public health

onde se lê:

mass event (ME)

leia-se:

mass gatherings (MG) 